\title{
A comparative FMADM method used to solve real life problem
}

\author{
Muley A.A. and Bajaj V.H.* \\ ${ }^{*}$ Department of Statistics, Dr. B. A. M. University, Aurangabad, MS, 431004, India \\ vhbajaj@gmail.com, aniket.muley@gmail.com
}

\begin{abstract}
In this paper, the concept based on entropy analysis is proposed. To determine the attribute weights under the conditions that the attribute weights are completely unknown. Furthermore, in order to make a decision or choose the optimum alternative, Using entropy analysis method attribute weights are completely known. To take a decision or choose optimal alternative we proposed the vertex distance method and calculate closeness for obtaining best ideal alternative solution. Compare this method using Additive Utility Function to check the feasibility and validity of the proposed study. Finally, a numerical example has been provided to illustrate the solution procedure on the proposed method.
\end{abstract}

Keywords- MADM, Triangular fuzzy number, Decision making, Entropy weight

\section{Introduction}

Multiple attribute decision making (MADM) is dealing with the problem of choosing the most desirable alternative that has the highest degree of satisfaction from a set of alternatives in regard to their attributes [1, 4, 6-7, 13-15]. There are complex MADM problems which involve both quantitative and qualitative attributes as well as various types of uncertainties. A fuzzy entropy measure is employed to partition the input feature space into decision regions and to select relevant features with good separability for the classification task. The proposed feature selection method based on the fuzzy entropy increases the classification rate by discarding noise-corrupted, redundant, and unimportant features. Firstly, we use a fuzzy entropy measure instead of fuzzy rules to reflect the actual distribution of classification patterns by computing the fuzzy entropy of each feature dimension. The decision region can be tuned automatically according to the fuzzy entropy measure. We propose an efficient method for selecting relevant features based on fuzzy entropy. Our proposed fuzzy entropy is derived from Shannon's entropy [12]. This concept has been defined in various ways and generalized in different applied fields, such as Communication theory, Mathematics, Statistical thermodynamics, and Economics [5, 9, 11-12]. Shannon contributed the broadest and the most fundamental definition of the entropy measure in Information theory. Entropy is a measure of the amount of uncertainty in the outcome of a random experiment, or a measure of the information obtained when the outcome is observed. The proposed method is based on entropy analysis to determine the attribute weights under the conditions that the attribute weights are completely unknown. The variables in decision matrix are expressed by means of triangular fuzzy numbers. We construct an optimization model by transforming the utility for each alternative into a real-valued function. Furthermore, in order to make a decision or choose the optimum alternative, an entropy analysis method is presented under the assumption that attribute weights are known fully. Then Vertex distance method is proposed to calculate closeness of best ideal alternative solution. The present study has only precise values for the performance rating and for the attribute weights which are calculated from subjective approach. After that, additive utility function is used and considers subjective weights calculated from the above model to obtain best alternative solution. Finally, a numerical example is taken to further verify the validity and the feasibility of the proposed method.

\section{The fuzzy MADM problem}

In this paper, the triangular membership function has been used and it is defined as follows:

Definition 1: The triangular fuzzy number $\AA^{c}$ is defined as combination of $(a, b, c)$, its relative function is defined as Zimmermann [16]:

$$
\mu_{\not \circ}(x)= \begin{cases}0, & x<a \\ \frac{x-a}{b-a}, & a \leq x \leq b \\ \frac{c-x}{c-b}, & x>c\end{cases}
$$

Definition 2.: Let $A^{\prime}$ and $B^{\prime}$ are two positive triangular fuzzy number represent by

combination $\left(a_{l}, a_{m}, a_{u}\right)$ and $\left(b_{l}, b_{m}, b_{u}\right)$, their operational laws are represented as Chang [2]:

1. $a \oplus b=\left(a_{l}, a_{m}, a_{u}\right) \oplus\left(b_{l}, b_{m}, b_{u}\right)=\left(a_{l} \oplus b_{l}, a_{m} \oplus b_{m}, a_{u} \oplus b_{u}\right) ;$
2. $a \otimes b=\left(a_{l}, a_{m}, a_{u}\right) \otimes\left(b_{l}, b_{m}, b_{u}\right)=\left(a_{l} b_{l}, a_{m} b_{m}, a_{l} b_{u}\right) ;$
3. $\lambda \otimes a=(\lambda, \lambda, \lambda) \otimes\left(a_{l}, a_{m}, a_{u}\right) \approx\left(\lambda a_{l}, \lambda a_{m}, \lambda a_{u}\right) ;$
4. $\frac{1}{a} \approx\left(\frac{1}{a_{l}}, \frac{1}{a_{m}}, \frac{1}{a_{u}}\right)$.
Suppose $A=\left(A_{1}, A_{2}, \ldots, A_{n}\right)$ is a discrete set of $n(n>2)$ potential alternatives, where the alternatives are known and $\mathrm{N}=(1,2, \ldots, n)$. Let $C=\left(C_{1}, C_{2}, \ldots, C_{m}\right)$ be the set of attributes, and $w=\left(w_{1}, w_{2}, \ldots, w_{m}\right)$ be the weight vector of attributes in which the attribute weights are completely unknown, such that $w_{i}$ is an exact 
numerical value, and $\sum_{i=1}^{m} w_{i}=1, w_{i} \geq 0, i \in M=\{1,2, \ldots, m\}$. For the alternative $A_{j} \in A$ with respect to the attribute $C_{j} \in C, \quad x_{i j}$ is a fuzzy MADM problem can be concisely expressed in matrix format called fuzzy decision matrix $X=\left(x_{i j}\right)_{m \times n}$ as

$A_{1} \quad A_{2} \quad \ldots \quad A_{n}$

$C_{1}\left[\begin{array}{llll}x_{11} & x_{12} & \ldots & x_{1 n}\end{array}\right.$

$\begin{array}{lllll}C_{2} & x_{21} & x_{22} & \ldots & x_{2 n}\end{array}$

.. $\ldots \quad \ldots \quad \ldots \quad \ldots$

$C_{m}\left[\begin{array}{llll}x_{m 1} & x_{m 2} & \ldots & x_{m n}\end{array}\right]$

$\gamma_{g}=\frac{x_{y}}{\left\|x_{s}\right\|} \quad$ for benifitattribute $C_{t}$

Where, $x_{i j}=\left(x_{l i j}, x_{m i j}, x_{u i j}\right)$ is a triangular fuzzy variable.

$r_{i j}=\frac{1 / x_{4}}{\left\|1 / x_{i}\right\|} \quad$ for cost attribute $C_{i}$

Usually, there are two categories of attribute including benefit attribute and cost attribute in the MADM problem. In order to eliminate the incommensurability of the attributes in dimensionless units, each attribute $x_{i j}$ in the matrix $\quad X=\left(x_{i j}\right)_{m \times n}$ needs to be normalized into the corresponding comparable element in the matrix $R=\left(r_{i j}\right)_{m \times n}$, by using the following formulas where $r_{i j}=\left(r_{l i j}, r_{m i j}, r_{u i j}\right), i \in M, j \in N$, and $\left\|x_{i}\right\|=\sqrt{\sum_{j=1}^{n} x_{i j}^{2}}, \quad\left\|\frac{1}{x_{i}}\right\|=\sqrt{\sum_{j=1}^{n}\left(\frac{1}{x_{i j}}\right)^{2}}$.

From formula (2) and (3), according to the operational laws of triangular fuzzy variables we can obtain the following conclusions:

$$
\begin{aligned}
& r_{l i j}=\frac{x_{l i j}}{\sqrt{\sum_{j=1}^{n} x_{u}^{2}}} \\
& r_{m i j}=\frac{x_{m i j}}{\sqrt{\sum_{j=1}^{n} x_{m}^{2}}} \\
& r_{u i j}=\frac{x_{u i j}}{\sqrt{\sum_{j=1}^{n} x_{l i j}^{2}}} \\
& \text { for benefit attribute } C_{i}, i \in M, j \in N \text {, } \\
& r_{l i j}=\frac{\left(1 / x_{u i j}\right)}{\sqrt{\sum_{j=1}^{n}\left(1 / x_{l i j}^{2}\right)}} \\
& r_{m i j}=\frac{\left(1 / x_{m i j}\right)}{\left.\sqrt{\sum_{j=1}^{n}\left(1 / x_{m i j}^{2}\right.}\right)} \\
& r_{u i j}=\frac{\left(1 / x_{l i j}\right)}{\sqrt{\sum_{j=1}^{n}\left(1 / x_{u i j}^{2}\right)}}
\end{aligned}
$$

for cost attribute $C_{i}, i \in M, j \in N$.

Then the fuzzy MADM addresses the problem of ranking alternatives or choosing the most desirable alternative among the finite set of alternatives based on the normalized decision matrix $R=\left(r_{i j}\right)_{m \times n}$.

\section{Entropy based Ideal solution Method}

Here, proposed method is to calculate the precise performance rating to an alternative for the consideration.

Definition 3. A fuzzy set $\mathscr{G} /$ in a universe of discourse $X$ is characterized by a membership function $\mu_{a \delta}(x)$ which associates with each element $x$ in $X$, a real number in the interval $[0,1]$. The function value is termed the grade of membership of $x$ in $\mathscr{G} /$ Yang and Chou [15].The present study uses triangular fuzzy numbers. A triangular fuzzy number $\mathscr{V}_{1}$ can be defined by a triplet $\left(a_{l}, a_{m}, a_{u}\right)$. Its conceptual schema and mathematical form are shown below; Zimmermann [16], 
$\mu_{a O}(x)= \begin{cases}0, & x \leq a_{l}, \\ \frac{x-a_{l}}{a_{m}-a_{l}}, & a_{l} \leq x \leq a_{l}, \\ \frac{a_{u}-x}{a_{m}-a_{l}}, & a_{m} \leq x \leq a_{u}, \\ 0, & x \geq a_{u},\end{cases}$

Definition 4. Let $\mathscr{d}=\left(a_{l}, a_{m}, a_{u}\right)$ and $b^{\circ}=\left(b_{l}, b_{m}, b_{u}\right)$ be two triangular fuzzy numbers, then the vertex method is defined to calculate the distance between them,

$d(a b b)=\sqrt{\left(a_{1}-b_{1}\right)^{2}+\left(a_{2}-b_{2}\right)^{2}+\left(a_{3}-b_{3}\right)^{2}}$

(4)

Assuming that both $\mathscr{d} \in=\left(a_{l}, a_{m}, a_{u}\right)$ and $b=\left(b_{l}, b_{m}, b_{u}\right)$ are real numbers, then the distance measurement $d(a d b)$ is identical to the Euclidean distance by Chen [3].

Then normalized fuzzy decision matrix is calculated by using Eq. (2)-(3) can be represented as below

$$
R^{Q / O}=[\%]_{m \times n}
$$

The weighted fuzzy normalized decision matrix is shown as Eq. (6)

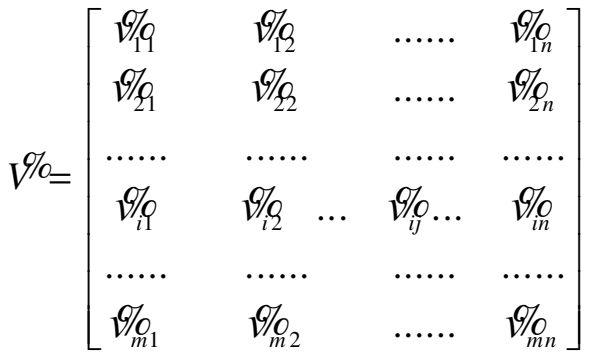

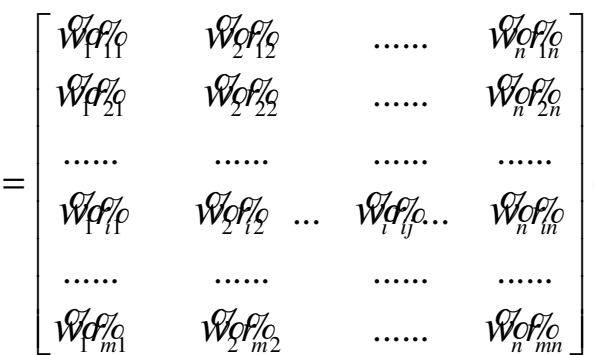

Given the above fuzzy theory, the proposed method is defined as follows:

Step 1: Choose the triangular rating $(X O l j, i$

$=1,2, \ldots, m, j=1,2, \ldots, n)$ for alternatives with respect to criteria and the weights are consider from the entropy analysis $\left.W_{j}, j=1,2, \ldots, n\right)$ to the criteria. Shannon's Entropy [12], which demonstrates that a broad distribution represents more ambiguity than does a sharply peaked one is applied to determine the objective weight in our study. As we know, entropy theory is an important theory to study the problem of uncertainty. Entropy weight is a parameter that describes how much different alternatives approach one another in respect to certain attribute. The greater the value of the entropy, the smaller the entropy weight, then the smaller the differences of different alternatives in this specific attribute, and the less information the specific attribute provides, and less important this attribute becomes in the decision making process.

For crisp numbers, the calculation of the entropies is very straightforward. Usually we will use the following formula:

$$
E(i)=-K \sum_{i=1}^{m} x_{i j} \ln x_{i j} \text {, (7) }
$$

Where $K$ is a constant.

While for fuzzy numbers, we can not use the above formula to calculate the entropies of fuzzy numbers directly. Generally, we would first transform the fuzzy numbers into crisp numbers, and then calculate their respective entropies. Although there are many methods to transform fuzzy numbers, most of these methods did not take into account the decision maker's preferences for the degree of uncertainties. A formula that took into account these factors:

$$
\begin{aligned}
& F\left(r_{i j}\right)=m\left(r_{i j}\right)-\beta \sigma\left(r_{i j}\right)(8) \\
& m\left(r_{i j}\right)=\frac{\int r u\left(r_{i j}\right) d r}{\int u\left(r_{i j}\right)},(9) \\
& \sigma\left(r_{i j}\right)=\left[\frac{\int r^{2} u\left(r_{i j}\right) d r}{\int u\left(r_{i j}\right)}-m^{2}\left(r_{i j}\right)\right]^{1 / 2} \\
& )
\end{aligned}
$$

If $x_{i j}$ is triangle, then formula (6) and (7) by Lee and $\mathrm{Li}[10]$ can be simplified as:

$$
\begin{aligned}
& m\left(r_{i j}\right)=\frac{1}{3}(l+m+n),(11) \\
& \sigma\left(r_{i j}\right)=\frac{1}{18}\left(l^{2}+m^{2}+n^{2}-l m-l n-l m\right)
\end{aligned}
$$

(12)

and $I=$ lower, $m=$ middle and $u=$ upper, of

fuzzy triangular number $r_{i j}$.

Where $F\left(r_{i j}\right)$ represents the ranking index of the $j^{\text {th }}$ attribute of the $i^{\text {th }}$ alternative, $\beta$ represents the decision maker's uncertaintyaversion coefficient, when $\beta>0$, the decision maker is uncertainty-averse; when $\beta<0$, the decision maker is loving; and when $\beta=0$, the decision maker is uncertainty neutral. Next, normalize $F\left(x_{i j}\right)$ according to the following equation: 


$$
f_{i j}=\frac{F\left(r_{i j}\right)}{\sum_{i=1}^{m} F\left(r_{i j}\right)}
$$

Then, the fuzzy entropies of the attributes can be calculated with the following:

$$
E_{j}^{O}=-K \sum_{i=1}^{m} f_{i j} \ln f_{i j}=-\frac{1}{\ln m} \sum_{i=1}^{m} f_{i j} \ln f_{i j}(14)
$$

Now, calculate the fuzzy entropy weight the following equation:

$$
w_{j}^{o}=\frac{1-E_{j}^{o}}{\sum_{i=1}^{m} 1-E_{j}^{o}}
$$

Step 2: Construct the weighted normalized fuzzy decision matrix. The weighted normalized value $V / \%$ is calculated by Eq. (6).

Step 3: Identify positive ideal $\left(A^{+}\right)$and negative ideal $\left(A^{-}\right)$solutions. The fuzzy positive ideal solution (FPIS, $A^{+}$) and the fuzzy negative ideal solution (FNIS, $A^{-}$) are shown as Eqs. (16) and (17):

$$
\begin{aligned}
A^{+} & =\left(v_{1}^{+}, v_{2}^{+}, \ldots, v_{n}^{+}\right) \\
& =\left\{\max v_{i j} \mid i=\underset{j}{j} \underset{j}{2, \ldots, m, j=1,2, \ldots, n\}}\right. \\
A^{-} & =\left(v_{1}^{-}, v_{2}^{-}, \ldots, v_{n}^{-}\right) \\
& =\left\{\max v_{i j} \mid i=1,2, \ldots, m, j=1,2, \ldots, n\right\}
\end{aligned}
$$

Step 4: Calculate separation measures. The distance of each alternative from $A^{+}$and $A^{-}$can be currently calculated using Eqs. (18) and (19):

$$
\begin{aligned}
& d^{+}=\sum_{j=1}^{n} d(1 / \overbrace{l j}, 1 /\left.\right|_{j} ^{+}), i=1,2, \ldots, m \text { (18) } \\
& d^{-}=\sum_{j=1}^{n} d\left(V / V_{l}, V / o\right), i=1,2, \ldots, m
\end{aligned}
$$

Step 5: Calculate similarities to ideal solution. This step solves the similarities to an ideal solution by Eq. (20):

$$
C C_{i}=\frac{d^{-}}{d^{+}+d^{-}}(20)
$$

Step 6: Rank preference order. Choose an alternative with maximum $\mathrm{CC}_{i}^{+}$or rank alternatives according to $\mathrm{CC}_{i}^{+}$in descending order.

We consider another method i.e. the additive utility function is proposed. This method is compared with the above proposed method. After calculating the utility values to determine the most preferred alternative or rank alternatives in descending order. Also we take entropy weight for calculating fuzzy utilities of the alternatives according to the following equation:

$$
U \%\left(A_{i}\right)=\sum_{j=1}^{n}\left(w_{j}^{o} \otimes r_{i j}\right)(21)
$$

The decision makers use the triangular variables to evaluate the importance of attributes and the rating of alternatives with respect to various attributes. Finally, we check the feasibility and validity of the Entropy based Ideal solution method with additive utility function method.

\section{Numerical example}

To illustrate the proposed method for the MADM problem a case study of solving distribution center location in logistics systems is taken. For the logistics system, location of distribution center is one of the most important components. In order to select the proper distribution center, a logistics company considers four possible alternatives denoted as $A_{1}, A_{2}, A_{3}, A_{4}$. Moreover, the main attributes

\begin{tabular}{|c|c|c|c|}
\hline & & & \\
\hline$(0.80,0.85,0.90)$ & $90,0.95,1.00)$ & $(0.88,0.91,0.95)$ & \\
\hline $0.92,0.95)$ & $39,0.90,0.93)$ & $84,0.8 \mathrm{z}-2$ & \\
\hline & . & , & \\
\hline & $(094,0$ & $(0.91,0$ & \\
\hline$(0.90,0.91,0.92)$ & $(0.90,0.92,0.95)$ & $0.92)$ & \\
\hline \multicolumn{4}{|c|}{$\begin{array}{l}\text { rst, we normalize the decision matrix } X \text { into } \\
e \text { corresponding matrix } R \text { by using the } \\
\text { rmulas (2) and (3), then the normalized } \\
\text { ecision matrix } R \text { is given in Table } 2 \text {. } \\
\text { Table } 2 \text { - Normalized decision matrix } R\end{array}$} \\
\hline$A_{1}$ & $A_{2}$ & & 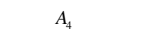 \\
\hline 42 & 0.47, & 46,0 . & \\
\hline \begin{tabular}{l|l}
$C_{2}$ & $(0.48,0.51,0$
\end{tabular} & $(0.48,0.50,0$ & $(0.45,0.48,0.51)$ & $(0.48,0.51$, \\
\hline$(0.47,0.5)$ & $0.47,0$ & $0.47,0$ & \\
\hline $0.40,0$ & $(0.48,0.51,0.5)$ & $(0.47,0.49,0.5$ & \\
\hline & & & \\
\hline
\end{tabular}
influencing distribution center location involve: $C_{1}$-service level, $C_{2}$-social benefit, $C_{3}$-natural environment, $C_{4}$-public infrastructure, $C_{5}$ - cost, where the attributes $C_{1}, C_{2}, C_{3}$ and $C_{4}$ are benefit attributes, and $C_{5}$ attribute is cost attribute. The fuzzy ratings of alternatives $A_{j},(j \in N) \quad$ according to attributes $C_{i},(i \in M)$ are evaluated by decision maker and form the decision matrix $X$ given below: Table 1- Decision matrix $X$

By using equation (8), let $\beta=2$, we get $F\left(r_{i j}\right)$ as:

Table 3

$$
F\left(r_{i j}\right)=\left[\begin{array}{llll}
0.4692 & 0.5255 & 0.5059 & 0.4957 \\
0.5097 & 0.5031 & 0.4797 & 0.5097 \\
0.4964 & 0.4931 & 0.5028 & 0.5062 \\
0.5097 & 0.5064 & 0.4931 & 0.4899 \\
0.4999 & 0.4931 & 0.5097 & 0.4897
\end{array}\right]
$$

Then calculating relative frequency $f_{i j}$ by equation (13), we get

Table 4 


$$
f_{i j}=\left[\begin{array}{llll}
0.2550 & 0.2632 & 0.2534 & 0.2483 \\
0.2546 & 0.2513 & 0.2396 & 0.2546 \\
0.2484 & 0.2467 & 0.2516 & 0.2533 \\
0.2549 & 0.2533 & 0.2466 & 0.2451 \\
0.2509 & 0.2475 & 0.2566 & 0.2458
\end{array}\right]
$$

After simplifying equation (13) and (14), we obtain the weight value $w_{i}^{o},(i \in M)$ as follows:

$w_{1}^{o}=0.2005, w_{2}^{o}=0.1999, w_{3}^{o}=0.1998, w_{4}^{o}=0.1999, w_{5}^{o}=0.1998$

Here, we used these entropy weights for solving our proposed method i.e. $w_{i}^{o},(i \in M)$. Then by Eq. (5) and multiplying corresponding weights of the criteria i.e. Eq. (6), weighted normalized matrix. we know that the elements $\quad \% i, \forall i, j$ are normalized positive triangular fuzzy numbers and their range belongs to the closed interval $[0,1]$. Thus, we can define the fuzzy positive ideal solution (FPIS, $A^{+}$) and the fuzzy negative solution (FNIS, $2>4>3>1$ ) as: ${ }_{j} F_{0}=(1,1,1)$ and $9 F_{j}=(0,0,0)$. This is third step of fuzzy analysis.

For the fourth step, After calculating separation measures this term the distance of each alternative from $A^{+}$and $A^{-}$can be currently calculated using Eqs. (19) and (20), we get;

$d_{1}^{+}=7.7989, \quad d_{2}^{+}=7.7862, \quad d_{3}^{+}=7.7967, \quad d_{4}^{+}=7.7968$

$d_{1}^{-}=0.8625, \quad d_{2}^{-}=0.8752, \quad d_{3}^{-}=0.8649, \quad d_{4}^{-}=0.8650$

Then Calculating similarities to ideal solution by Eq. (20):

$C C_{1}=0.09957, \quad C C_{2}=0.10105, \quad C C_{3}=0.09985, \quad C C_{4}=0.09986$

Therefore; we can rank all the alternatives as: $A_{2}>A_{4}>A_{3}>A_{1}$ and then the best alternative is $A_{2}$.

Now, the Additive Utility function is proposed. To simplify this function, weight vector $w^{o}=\left(w_{1}^{o}, w_{2}^{o}, \ldots, w_{5}^{o}\right)$ is taken from Eq. (15) and substitute into Eq. (21), we get the overall

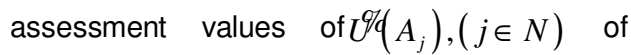
alternatives.

$$
\begin{aligned}
& U \%\left(A_{1}\right)=(0.4659,0.4979,0.5279) \\
& U \%\left(A_{2}\right)=(0.4739,0.5039,0.5359) \\
& U \%\left(A_{3}\right)=(0.4659,0.4979,0.5319) \\
& U \%\left(A_{4}\right)=(0.4659,0.4980,0.5319)
\end{aligned}
$$

Rank the alternatives using equation (8), let $\beta$ $=0$.

$$
\begin{gathered}
F\left(A_{1}\right)=0.4972, \quad F\left(A_{2}\right)=0.5046, \\
F\left(A_{3}\right)=0.4985, \quad F\left(A_{4}\right)=0.4986 .
\end{gathered}
$$

Therefore; we can rank all the alternatives $A_{j},(j \in N)$ as follows:

$A_{2}>A_{4}>A_{3}>A_{1}$ and then the best alternative is $A_{2}$.

\section{Conclusion}

Here, we have proposed the Entropy based Ideal solution method for the MADM problem, in which the preference values take the form of triangular fuzzy numbers, and attribute weight values are completely unknown. Entropy analysis of fuzzy variables is used to determine attribute weights. Then, additive utility function has been used for ranking alternatives. Then proposed approach has been used to select the location of distribution center in logistics systems. Among the four alternatives available the best alternative is found $A_{2}$. After comparing these two methods we get the same result i.e., the best alternative is found $A_{2}$. But as compared Entropy based Ideal solution method with additive utility function the proposed approach is more useful to solve the subjective closeness towards the problem. Entropy based Ideal solution method is used to increase the closeness to the best alternative selection. In future, one may use this method to develop large scale problem at various stages in industrial sector.

\section{References}

[1] Bellman R. E. and Zadeh L.A. (1970) Management Science, 17B (4), 141-164.

[2] Chang D. Y. (1996) European Journal of Operational Research, 95, 649-655.

[3] Chen C. T. (2000) Fuzzy Sets and Systems, $114,1-9$.

[4] Chen S.J. and Hwang, C.L. (1992) SpringerVerlag, Berlin.

[5] Cover T. M. and Thomas J. A. (1992) New York, Wiley.

[6] Deng H., Yeh C. H. \& Willis R. J. (2000) Computers and Operations Research, 27. 963-973.

[7] Hwang C.L., Yoon K. P. (1981) SpringerVerlag, Berlin.

[8] Hwang C. L and Masud A.S.M. (1979) Springer, Berlin.

[9] Kosko B. (1986) Information Science, 40, 165174.

[10] Lee E. S. and Li R. L. (1988) Computer and Mathematics with Application, 15, 887-896.

[11] Pal S. K. and Chakraborty, B. (1986) IEEE Trans. Syst., Man, Cybernetics SMC., 16, 754-760.

[12] Shannon C. E. (1948) Bell Syst. Tech. J., 27, 379-423.

[13] Wang Y.M., Parkan C. (2005) Fuzzy Sets and Systems, 153, 331-346.

[14] Xu Z. S. (2007) Knowledge- Based System, 20, 719-725.

[15] Yang T. and Chou P. (2005) Mathematics and Computer in Simulation, 68, 9-21.

[16] Zimmermann H. J. (1996) Kluwer Academic Publisher, Dordrecht. 